\title{
INFLUÊNCIA DA EXTENSÃO DA ARTRODESE LOMBOSSACRA NOS RESULTADOS CLIINICOS E FUNCIONAIS
}

\author{
INFLUENCE OF THE EXTENT OF LUMBOSACRAL ARTHRODESIS IN CLINICAL \\ AND FUNCTIONAL OUTCOMES
}

\author{
INFLUENCIA DE LA EXTENSIÓN DE LA ARTRODESIS LUMBOSACRA \\ EN LOS RESULTADOS CLÍNICOS Y FUNCIONALES
}

Alberto Ofenhejm Gotfryd', Guilherme Galito Henriques², Patrícia Rios Poletto ${ }^{3}$

\begin{abstract}
RESUMO
Objetivo: Verificar a influência da extensão da fusão póstero-lateral lombossacra e seu impacto nos resultados clínicos e funcionais. Métodos: Foram avaliados 22 pacientes portadores de estenose central, foraminal ou hérnia de disco lombar, associadas a instabilidade segmentar, submetidos ao tratamento cirúrgico para a descompressão neural e artrodese póstero-lateral. Os pacientes foram divididos em dois grupos, de acordo com quantidade de níveis artrodesados: Grupo 1: fusão em um nível e Grupo 2: fusão em dois ou mais níveis. Os pacientes preencheram questionários referentes ao acompanhamento pós-operatório (uso de medicamentos analgésicos e satisfação com o tratamento) e escala analógica visual de dor lombar e ciática. Além disto, foram aplicados os questionários Oswestry e SF-36 para avaliação da qualidade de vida. Resultados: Os resultados do SF-36 mostraram bons níveis de qualidade de vida em ambos os grupos, com exceção do domínio "Aspectos Físicos". Não houve correlação significativa entre a extensão da artrodese e os desfechos clínicos. Conclusões: Os pacientes submetidos à artrodese póstero-lateral apresentaram qualidade de vida satisfatória, exceto pela diminuição da aptidão física. Entretanto, o maior número de níveis artrodesados não teve impacto negativo sobre os aspectos clínicos e funcionais.
\end{abstract}

Descritores: Coluna vertebral; Dor lombar; Artrodese; Descompressão cirúrgica; Qualidade de vida; Ciática; Parafusos ósseos.

\begin{abstract}
Objective: Investigate the influence of the extent of posterolateral lumbosacral fusion and its impact on clinical and functional results. Methods: We evaluated 22 patients with central or foraminal stenosis or lumbar disc herniation associated with segmental instability that underwent to surgery for nerve decompression and posterolateral arthrodesis. Patients were divided into two groups according to the number of levels fused: Group 1: fusion at one level and Group 2: fusion of two or more levels. Patients completed questionnaires regarding postoperative follow-up (use of analgesic medications and treatment satisfaction) and visual analog scale for lumbar pain and sciatica. Moreover, the questionnaires Oswestry and SF-36 were administered to assess quality of life. Results: SF-36 showed good levels of quality of life in both groups, except the field "Physical Aspects". There was no significant correlation between the extent of the arthrodesis and clinical outcomes. Conclusions: Patients who underwent posterolateral arthrodesis showed satisfactory quality of life, except for a decrease of physical fitness. However, the higher number of levels fused did not have negative impact on clinical and functional aspects.
\end{abstract}

Keywords: Spine; Low back pain; Arthrodesis; Decompression surgical; Quality of life; Sciatica; Bone screws.

\section{RESUMEN}

Objetivo: Investigar la influencia del grado de la fusión posterolateral lumbosacral y su impacto en los resultados clínicos y funcionales. Métodos: Se evaluaron 22 pacientes con estenosis central, foraminal o hernia de disco lumbar, asociadas a inestabilidad segmentaria; se sometieron a cirugía para la descompresión neural y la artrodesis posterolateral. Los pacientes fueron divididos en dos grupos según el número de niveles fusionados: Grupo 1: la fusión en un nivel, y Grupo 2: la fusión en dos o más niveles. Los pacientes completaron cuestionarios sobre seguimiento posoperatorio (uso de medicamentos analgésicos y satisfacción con el tratamiento) y la escala analógica visual para el dolor lumbar y la ciática. Por otra parte, se aplicaran los cuestionarios Oswestry y SF-36 para evaluar la calidad de vida. Resultados: El SF-36 mostró resultados de buenos niveles de calidad de vida en ambos grupos, excepto en el campo "Aspectos Físicos". No hubo correlación significativa entre la magnitud de la artrodesis y los resultados clínicos. Conclusiones: Los pacientes que fueron sometidos a artrodesis posterolateral mostraron calidad de vida satisfactoria, con excepción de disminución de la aptitud física. Sin embargo, el mayor número de niveles fusionados no tuvo un impacto negativo en los aspectos clínicos y funcionales.

Descriptores: Columna vertebral; Dolor en la región Lumbar; Artrodesis; Descompresión quirúrgica; Calidad de vida; Ciática; Tornillos óseos.

1. Mestre, Médico Chefe do Grupo de Coluna Vertebral do Departamento de Ortopedia eTraumatologia da Santa Casa da Misericórdia de Santos - Santos, SP, Brasil.

2. Médico Ortopedista, Estagiário do Grupo de Coluna Vertebral do Departamento de Ortopedia eTraumatologia da Santa Casa da Misericórdia de Santos - Santos, SP, Brasil.

3. Doutor, Professor Adjunto do Curso de Fisioterapia da Universidade Federal de São Paulo - São Paulo, SP, Brasil.

Trabalho realizado no Departamento de Ortopedia eTraumatologia - Santa Casa da Misericórdia de Santos.

Correspondência: Av. Ana Costa 259 / 51, Encruzilhada - Santos - SP. Brasil. 11060-001. albertocoluna@yahoo.com.br 


\section{INTRODUÇÃO}

A incidência de doenças degenerativas da coluna vertebral têm aumentado nas últimas décadas em decorrência da crescente expectativa de vida populacional ${ }^{1,2}$. Estima-se que $53 \%$ dos brasileiros economicamente ativos irão apresentar lombalgia incapacitante ao longo de sua vida e $33 \%$ terão dor ciática associada 3 .

Apesar do tratamento conservador ter uma alta taxa de bons resultados ${ }^{4}$ e ser predominante nos casos de dor ciática secundária a hérnias discais ou estenose do canal vertebral, a cirurgia pode estar indicada quando não houver regressão do quadro álgico por pelo menos seis semanas, se persistência e/ou progressão do déficit neurológico e nos casos de crises repetitivas e incapacitantes de lombociatalgia ${ }^{4,5}$. O tratamento cirúrgico das afecções degenerativas lombossacras, quando acompanhadas de instabilidade mecânica, deve contemplar a melhora da dor e/ ou da disfunção neurológica (descompressão) e a estabilização mecânica (artrodese) ${ }^{6,7}$.

Apesar de vários estudos terem reportado a importância clínica de uma fusão intervertebral sólida ${ }^{8-13}$, persiste a controvérsia sobre o impacto da extensão da mesma (único ou múltiplos níveis) sobre a qualidade de vida destes doentes. A hipótese seria que, quanto maior a artrodese lombossacra piores seriam os escores de qualidade de vida e aptidão física.

O presente estudo teve por objetivo verificar a influência da extensão da fusão póstero-lateral lombossacra e seu impacto nos resultados clínicos e funcionais, em pacientes portadores de afecções degenerativas associadas a instabilidade mecânica.

\section{MATERIAIS E MÉTODOS}

Trata-se de um estudo transversal comparativo. Após aprovação no Comitê de ética em Pesquisa com Seres Humanos (número 52/10), os pacientes que preencheram os critérios de inclusão foram convidados a participar desta pesquisa e assinaram o Termo de Consentimento Livre e Esclarecido. Participaram do estudo 22 indivíduos portadores de estenose central, foraminal ou hérnia de disco associada a instabilidade mecânica (incluindo a primeira vértebra lombar ao sacro) que foram submetidos ao tratamento operatório no período compreendido entre março de 2007 e novembro de 2009. O tempo mínimo pós-operatório foi doze meses. Todas as cirurgias foram realizadas na Santa Casa da Misericórdia de Santos pelo mesmo cirurgião.

Os pacientes foram submetidos à descompressão neural através de discectomia e/ou foraminotomia ou laminectomia, auxiliada por magnificação de imagens com lupa de 2,5 vezes de aumento (Designs for Vision, Inc., NY, US), seguida de fusão póstero-lateral com a utilização de parafusos pediculares. Como rotina, foi utilizada enxertia autóloga, proveniente dos elementos posteriores locais (processos espinhosos, lâmina e facetas articulares) associada a fragmentos ósseos do ilíaco.

O detalhamento da indicação cirúrgica e os critérios de inclusão estão descritos na Figura 1. Considerou-se a presença de instabilidade mecânica nos casos de desequilíbrio do tronco no plano coronal ou sagital e espondilolisteses ou retrolisteses vistas nas radiografias laterais simples. Além disto, realizamos radiografias laterais ortostáticas em flexão e extensão máximas, com a finalidade de detectarmos instabilidade segmentar dinâmica nos níveis a serem descomprimidos. Utilizamos os critérios propostos Herkowitz ${ }^{14}$ e Muggleton et al. ${ }^{15}$, que incluem a presença de translação igual ou superior a $4 \mathrm{~mm}$ ou ângulação maior ou igual a 10 graus.

Os pacientes foram divididos em dois grupos de acordo com a quantidade de níveis artrodesados: Grupo 1 (um nível; N=7 pacientes), Grupo 2 (dois ou mais níveis; $\mathrm{N}=14$ pacientes). A qualidade da fusão foi avaliada pela radiografia ântero-posterior, segundo os critérios radiográficos propostos por Christensen et al. ${ }^{16}$. Foram preenchidos questionários referentes ao quadro clínico, uso de medicamentos analgésicos e satisfação pessoal. Além disto,

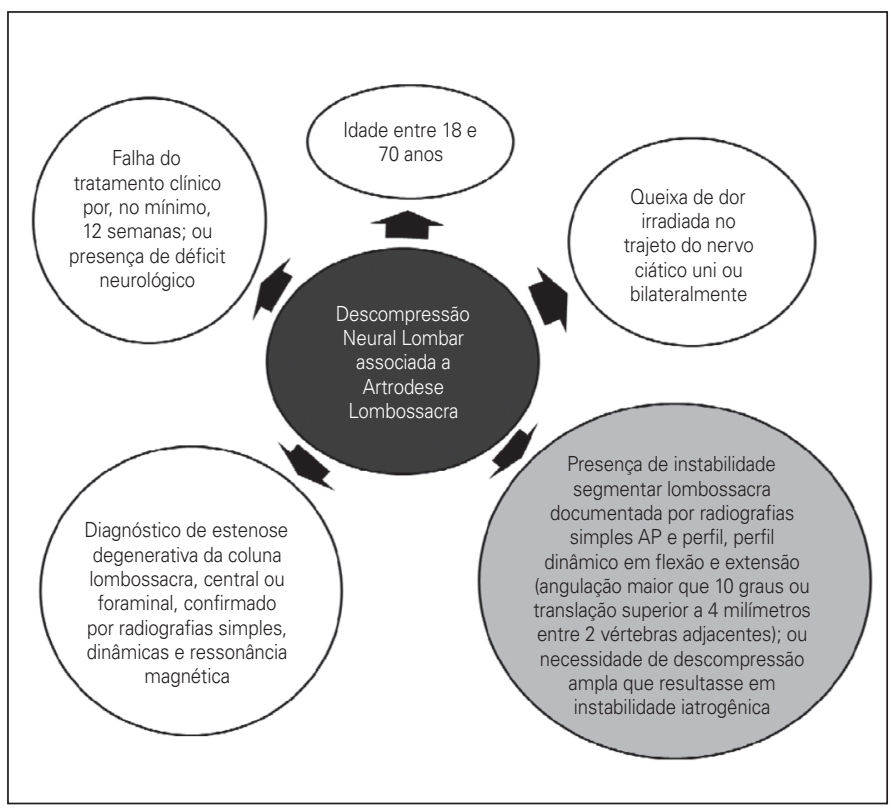

Figura 1. Critérios de inclusão e indicações cirúrgicas para descompressão neural lombar associada a artrodese lombossacra.

utilizamos a escala numérica de dor para quantificarmos a intensidade da dor lombar e ciática e os questionários Oswestry ${ }^{17}$, que avalia a incapacidade Funcional e SF-36 ${ }^{18}$, destinado à avaliação da qualidade de vida.

Os dados foram analisados com o programa estatístico SPSS versão 17.0 (SPSS Inc.,Chicago, IL, USA). A análise descritiva é mostrada em média e desvio padrão e porcentagem. As variáveis foram testadas quanto a sua normalidade pelo teste Kolmogorov-Smirnov e como não apresentaram distribuição normal foi utilizado o teste Mann-Whitney para verificar a diferença entre os grupos. Para todas as análises foi usado nível de significância de 0,05.

\section{RESULTADOS}

A amostra foi composta predominantemente por homens (76\%). Em relação à distribuição por idade, 67\% tinha menos de 60 anos. Os participantes apresentavam os seguintes diagnósticos pré-operatórios: estenose foraminal associada a espondilolistese/retrolistese $(48 \%)$, hérnia discal e instabilidade segmentar (33\%), estenose central degenerativa (14\%). A classificação de fusão indicou que $81 \%$ da amostra apresentou fusão sólida e 19\% fusão duvidosa. Dos 22 pacientes da amostra inicial, um apresentava pseudoartrose e foi excluído da amostra.

Na Figura 2 ainda pode-se observar a distribuição de porcentagens do uso de medicamentos.

Pode-se perceber na Figura 2 que a maioria da amostra estudada não faz mais uso de nenhum medicamento para dor lombar e/ou ciática. Na Tabela 1 podem ser observados os resultados descritivos que caracterizam a amostra: tempo de pós-operatório, idade, VAS, SF-36 e Oswestry.

Os resultados do SF-36 mostraram bons níveis de qualidade de vida, com exceção do domínio "Aspectos Físicos" que apresentou resultados regulares, especialmente para o Grupo 1. Em relação ao Questionário Oswestry os valores demonstraram pouco impacto da dor nas atividades diárias. A avaliação da escala numérica de dor mostrou baixos níveis de dor ciática para os dois grupos, dor lombar variando de leve a moderada no Grupo 2 e leve a intensa no Grupo 1, de acordo com a classificação da escala de dor de Breivk et al. ${ }^{19}$

Não houve diferença estatisticamente significativa entre os grupos para todas as variáveis apresentadas na Figura 2 e Tabela 1 $(p=0.100$ a 0.999). 


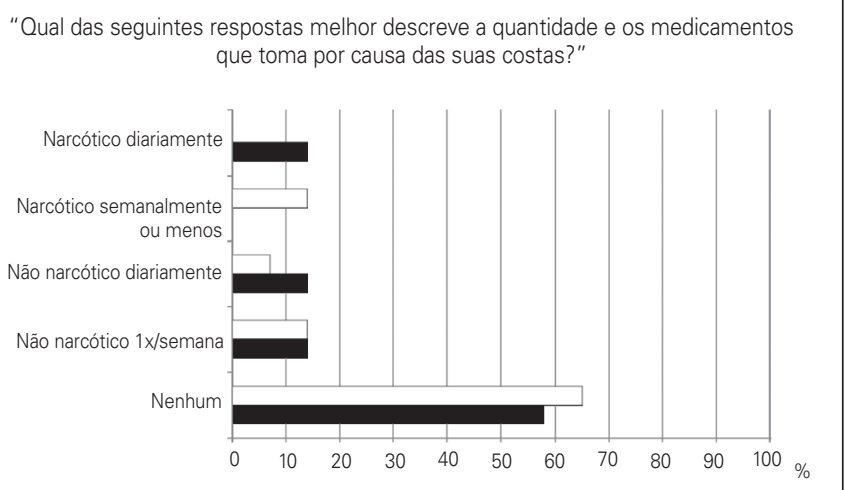

Figura 2. Distribuição por porcentagem da amostra de acordo com o uso de medicamentos.

Tabela 1. Caracterização da amostra estudada de acordo com a quantidade de níveis artrodesados.

\begin{tabular}{l|c|c}
\hline & Grupo 1 & Grupo 2 \\
\hline Tempo Pós-op (meses) & Média \pm dp & Média \pm dp \\
\hline Idade (anos) & $18 \pm 6$ & $25 \pm 8$ \\
\hline Intensidade Dor Lombar & $52 \pm 10$ & $59 \pm 12$ \\
\hline Intensidade Dor Ciática & $3 \pm 4$ & $3 \pm 3$ \\
\hline SF36 Domínio "Dor" & $1 \pm 2$ & $1 \pm 2$ \\
\hline SF36 Domínio "Capacidade Funcional" & $59 \pm 24$ & $59 \pm 27$ \\
\hline SF36 Domínio "Aspectos Físicos" & $36 \pm 45$ & $63 \pm 21$ \\
\hline SF36 Domínio "Aspectos Emocionais" & $71 \pm 49$ & $56 \pm 49$ \\
\hline Questionário Oswestry & $22 \pm 18$ & $24 \pm 17$ \\
\hline
\end{tabular}

\section{DISCUSSÃO}

Nosso estudo avaliou a influência da extensão da fusão lombossacra nos resultados clínicos e funcionais, em pacientes portadores de afecções degenerativas. Comparamos os resultados de pacientes submetidos a um nível de fusão àqueles com dois ou mais níveis. Hipoteticamente, quanto maior a quantidade de níveis artrodesados maiores seriam as limitações funcionais.

Os grupos estudados foram homogêneos em relação à idade, gênero e tempo de pós-operatório ( $p>0,05)$. O tempo mínimo de pós operatório considerado para a inclusão foi de doze meses, sendo a média para o Grupo 1 de 18 meses e para o Grupo 2 de 25 meses. Para a avaliação da qualidade de vida, utilizamos os questionários SF-36 e Oswestry, validados para a língua portugue$\mathrm{sa}^{17,18}$. Ambos forneceram informações importantes em relação à dor, capacidade funcional e aos aspectos físicos, além do impacto da dor nas atividades diárias destes indivíduos. Ainda, a Escala Numérica de Dor forneceu dados sobre a intensidade de dor lombar e ciática, tornando nossa metodologia multidimensional, característica importante na avaliação de enfermidades crônicas ${ }^{19}$.

A qualidade da fusão obtida é uma informação importante para o cirurgião de coluna. Apesar de autores como Kant et al. ${ }^{20}$ defenderem a exploração cirúrgica como método "padrão-ouro" para avaliação da massa de fusão, discordamos por considera- lo demasiadamente invasivo. Utilizamos os critérios propostos por Christensen et al. ${ }^{16}$, baseado em radiografias ântero-posteriores e que classifica o trabeculado ósseo intertransverso em: fusão completa, fusão duvidosa e pseudoartrose. Além do baixo custo, a técnica apresenta boa confiabilidade "intra" e "inter" avaliadores (93\% e $86 \%$ respectivamente). Segundo o método, $81 \%$ dos casos foram classificados como "fusão completa" e 19\% como "fusão duvidosa". O principal motivo que levou à dúvida sobre a consolidação foi a impossibilidade da visibilização adequada do leito de artrodese, devido à presença das hastes metálicas radiopacas. Excluímos 1 caso da amostra inicial, classificados como "pseudoartrose" e que foi considerado "outlier".

Sobre a qualidade da fusão, nossos resultados foram melhores do que os de Andersen et al. ${ }^{9}$, semelhantes aos de Dimar 2nd et al. ${ }^{13}$ e inferiores aos de Avanzi et al. ${ }^{8}$. Essas discrepâncias podem ser atribuídas aos diferentes critérios de classificação da qualidade da fusão usados nos estudos citados.

Outro aspecto clínico verificado em nosso estudo foi o uso de medicações analgésicas. Identificamos que a maioria dos pacientes dos dois grupos não faz uso de nenhum medicamento para alívio de dor lombar e/ou ciática. Encontramos três pacientes (14\%) que fazem uso de narcóticos ocasional ou regularmente. Este resultado é menor do que o de Dimar 2nd et al. ${ }^{13}$, que encontrou 32\% de uso de narcóticos em seu estudo, que avaliou aspectos clínicos e funcionais a curto, médio e longo prazo de pacientes submetidos à artrodese lombar.

De forma geral, foram encontrados bons níveis de qualidade de vida em nossa amostra, tanto para os indivíduos com um nível de artrodese quanto para aqueles com dois ou mais níveis. Os meIhores resultados foram verificados por meio dos domínios "Dor", "Aspectos Emocionais" e "Capacidade Funcional" do questionário SF-36. Já para o domínio "Aspectos Físicos", os resultados foram regulares, o que pode ser atribuído a uma possível limitação física. Entretanto, não encontramos diferenças neste domínio entre os grupos estudados, sugerindo que a maior extensão da fusão não tenha levado a piores desfechos clínicos. Throckmorton et al. ${ }^{21}$ encontraram resultados semelhantes aos nossos para os domínios "Dor", "Capacidade Funcional" e "Aspectos Físicos" do SF-36, ao avaliar pacientes submetidos `a descompressão neural associada a fusão em dois anos de seguimento, demonstrando boa qualidade de vida após a intervenção cirúrgica. Ainda em relação ao domínio "Aspectos Físicos", resultados semelhantes aos nossos foram observados nos estudos de Andersen et al. ${ }^{9} \mathrm{e}$ Dimar 2nd et a/ ${ }^{13}$.

O questionário Oswestry nos dá o indicativo do impacto da dor lombar e/ou ciática nas atividades diárias. No presente estudo obtivemos excelentes resultados neste questionário para os dois grupos, mostrando pouco impacto da dor e boa capacidade funcional. O mesmo foi verificado por Glassman et al. ${ }^{11}$ e por Berg et $\mathrm{al} .{ }^{22}$ em estudos com pacientes submetidos à descompressão neural associada à fusão.

Encontramos baixos níveis de dor lombar nos dois grupos avaliados. Nossos resultados são semelhantes aos de Glassman et al. ${ }^{11}$, Berg et al. ${ }^{22}$ e Valesin Filho et al. ${ }^{23}$. Para a intensidade da dor ciática residual, os valores foram baixos em ambos os grupos, evidenciando que o objetivo inicialmente proposto (descompressão neural) foi atingido e que os resultados são compatíveis com publicações prévias ${ }^{11,22}$.

Sobre as limitações de nosso estudo citamos o reduzido tamanho da amostra analisada, impossibilitando a análise de sub-grupo de acordo com o número de níveis anatômicos operados. Além disto, o fato de não termos registrado a avaliação pré-operatória completa destes índices para todos os pacientes nos impossibilitou de analisar as diferenças pré e pós operatórias. Por fim, o seguimento destes pacientes e a realização de futuros estudos poderão responder à esta questão clínica tardiamente.

\section{CONCLUSÃO}

Os pacientes submetidos à artrodese póstero-lateral apresentaram qualidade de vida satisfatória exceto pela diminuição da aptidão física. Entretanto, o maior número de níveis artrodesados não teve impacto negativo sobre os aspectos clínicos e funcionais. 


\section{REFERÊNCIAS}

1. Schwab FJ, Nazarian DG, Mahmud F, Michelsen CB. Effects of spinal instrumentation on fusion of the lumbosacral spine. Spine (Phila Pa 1976). 1995;20(18):2023-8.

2. Mooney V. Differential diagnosis of low back disorders: principles of classification. In Frymoyer JW, eds. The adult spine principles and practice. New York: Raven Press; 1991:1559-60.

3. Cecin AH, Molinar MHC, Borges MA, Morickochi M, Freire M, Bichuetti JAN. Dor lombar e trabalho: um estudo sobre a prevalência de lombalgia e lombociatalgia em diferentes grupos ocupacionais. Rev Bras Reumatol. 1991;31:50-6.

4. Iguchi T, Kanemura A, Kasahara K, Sato K, Kurihara A, Yoshiya S, et al. Lumbar instability and clinical symptoms: which is the more critical factor for symptoms: sagittal translation or segment angulation? J Spinal Disord Tech. 2004;17(4):284-90.

5. Gotfryd A, Avanzi O. A systematic review of randomised clinical trials using posterior discectomy to treat lumbar disc herniations. Int Orthop. 2009;33(1):11-7.

6. Hennemann SA, Schumacher W. Hérnia de disco lombar: revisão de conceitos atuais. Rev Bras Ortop. 1994; 29(3): 115-26.

7. Resnick DK, Choudhri TF, Dailey AT, Groff MW, Khoo L, Matz PG, et al. Guidelines for the performance of fusion procedures for degenerative disease of the lumbar spine. Part 8: lumbar fusion for disc herniation and radiculopathy. J Neurosurg Spine. 2005;2(6):673-8.

8. Avanzi O, Lin YC, Meves R, Silber MF. Tratamento da instabilidade lombar com parafusos pediculares. Acta Ortop Bras. 2005; 13(1):5-8

9. Andersen T, Videbaek TS, Hansen ES, Bünger C, Christensen FB. The positive effect of posterolateral lumbar spinal fusion is preserved at long-term follow-up: a RCT with 11-13 year follow-up. Eur Spine J. 2008;17(2):272-80.

10. Gibson S, McLeod I, Wardlaw D, Urbaniak S. Allograft versus autograft in instrumented posterolateral lumbar spinal fusion: a randomized control trial. Spine. 2002;27(15):1599-603.

11. Glassman SD, Carreon LY, Dimar JR, Campbell MJ, Puno RM, Johnson JR. Clinical out comes in older patients after posterolateral lumbar fusion. Spine J. 2007;7(5):547-51.

12. Bono $\mathrm{CM}$, Lee $\mathrm{CK}$. The influence of subdiagnosis on radiographic and clinical outcomes after lumbar fusion for degenerative disc disorders: an analysis of the literature from two decades. Spine (Phila Pa 1976). 2005 Jan 15;30(2):227-34.
13. Dimar JR 2nd, Glassman SD, Burkus JK, Pryor PW, Hardacker JW, Carreon LY. Two-year fusion and clinical outcomes in 224 patients treated with a single-level instrumented posterolateral fusion with iliac crest bone graft. Spine J. 2009;9(11):880-5.

14. Herkowitz HN. Spine update. Degenerative lumbar spondylolisthesis. Spine 1995;20(9):1084-90.

15. Muggleton JM, Kondracki M, Allen R. Spinal fusion for lumbar instability: does it have a scientific basis? J Spinal Disord. 2000;13(3):200-4.

16. Christensen FB, Laursen M, Gelineck J, Eiskjaer SP, Thomsen K, Bünger CE. Interobserver and intraobserver agreement of radiograph interpretation with and without pedicle screw implants: the need for a detailed classification system in posterolateral spinal fusion. Spine (Phila Pa 1976). 2001;26(5):538-43.

17. Vigatto R, Alexandre NM, Correa Filho HR. Development of a Brazilian Portuguese version of the Oswestry Disability Index: cross-cultural adaptation, reliability, and validity. Spine (Phila Pa 1976). 2007;32(4):481-6.

18. Ciconelli RM, Ferraz MB, Santos W, Meinão I, Quaresma MR. Tradução para a língua portuguesa e validação do questionário genérico de avaliação de qualidade de vida SF-36 (Brasil SF-36). Rev Bras Reumatol. 1999; 39:143-50.

19. Breivik H, Borchgrevink PC, Allen SM, Rosseland LA, Romundstad L, Hals EK, Kvarstein G, et al. Assessment of pain. Br J Anaesth. 2008;101(1):17-24.

20. Kant AP, Daum WJ, Dean SM, Uchida T. Evaluation of lumbar spine fusion. Plain radiographs versus direct surgical exploration and observation. Spine (Phila Pa 1976) 1995:20(21):2313-7.

21. Throckmorton TW, Hilibrand AS, Mencio GA, Hodge A, Spengler DM. The impact of adjacent level disc degeneration on health status outcomes following lumbar fusion. Spine (Phila Pa 1976). 2003;28(22):2546-50.

22. Berg S, Tullberg T, Branth B, Olerud C, Tropp H. Total disc replacement compared to lumbar fusion: a randomised controlled trial with 2-year follow-up. Eur Spine J. 2009;18(10):1512-9.

23. Valesin Filho ES, Ueno FH, Cabral LTB, Yonezaki AM, Nicolau RJ, Rodrigues LMR. Estudo prospectivo de avaliação de dor e incapacidade de pacientes operados de estenose de canal lombar com seguimento mínimo de dois anos. Coluna/Columna. 2009; 8(4):390-4 